\title{
A HIGH EFFICIENCY DC BUS REGULATOR / RPC FOR SPACECRAFT APPLICATIONS
}

\author{
Arthur G. Birchenough \\ lower and On-Board Propulsion T'echnology Division. NASA Glenn \\ Research Center at Lewis Field, Cleveland, Ohio 44135 \\ 216-433-6331, a.birchenoughanasa.gon
}

\begin{abstract}
DC bus voltage regulation may be required in future high powered spacecraft due to the length of the busses or because they are not generated at precise voltage levels. In these cases the regulation range is often only a few percent increase or decrease, but conventional DC voltage regulators switch all the power passing through them, and this level of power switched determines the size and losses in the regulator. A recently developed concept uses a low power DC-DC converter in series with the bus to raise or lower the bus voltage over a small range. This partial power processing technique combines the small size and power losses of the low power converter with the ability to regulate, (over a small range) a high power bus. The Series Connected Buck Boost Regulator (SCBBR) described herein provides bus regulation with an efficiency of $98 \%$. The circuit also provides bus switching and overcurrent limiting functions of a Remote Power Controller (RPC). This paper describes the circuit design and performance of a breadboard SCBBR configured as a bus voltage regulator providing $+/-40 \%$ voltage regulation range, bus switching, and overload limiting.
\end{abstract}

\section{INTRODUCTION}

The Brayton cycle power conversion system concept (Mason, 2003) for a future spacecraft will have an AC primary power distribution bus directly powering several high power loads, and a 120 volt DC regulated bus for the lower power loads. The power conversion for some of these high power loads and the DC bus is expected to be a transformer - rectifier -filter (TRF) set, potentially followed by a post regulator to provide for greater accuracy and regulation. The Series Connected Buck Boost Regulator (SCBBR) is presented as an option for providing high efficiency lightweight regulation and a current limiting / remote power controller (RPC) function on the DC bus.

DC-DC converter efficiency, size, and weight have improved steadily due to the improvements in semiconductors, and to a lesser extent due to improved passive components, topologies, and soft switching schemes. But these gains are gradual and evolutionary, not large step improvements. The change from linear to switching regulators was revolutionary, and the power loss and therefore size of regulators made a large step decrease. Since then the single most significant parameter determining converter efficiency and size, for a given frequency, has been the kva rating. The size of components to switch, transform, and filter is relatively independent of the topology, as the same amount of power is switched, rectified, and stored in filter components. However, the technique of partial power processing, wherein only a small fraction of the total output power is required to buck or boost the input to the desired output voltage, can significantly reduce the converter size and power loss.

The boost mode of the partial power processing technique has been used previously, wherein the low voltage output of a small DC-DC converter is connected in series with the input voltage (Button, 1996, 1998). The output voltage can then be adjusted from essentially equal to the input voltage up to the input voltage plus the maximum output 
voltage of the DC-DC converter. For example, a regulator for a 100 volt $1 \mathrm{~kW}$ bus could be constructed with a DCDC converter having a 100 volt nominal input, and a 0 to 10 volt output and a 100 watt, $10 \mathrm{amp}$, rating. The configuration would allow regulating the output to up to $10 \%$ higher than the input, and yet only use switching and filtering components sized for $10 \%$ of the total rating. Therefore the size and power loss are greatly reduced. The technique is very useful in situations where the input / output voltage ratio is relatively small, the output is always greater than the input. Also, this technique does not provide galvanic isolation between the input and output.

The series connected buck boost technique discussed in this paper expands the array of applications considerably by allowing the output to be higher or lower than the input. This allows essentially twice the regulation range for a given size converter. Additionally, a circuit enhancement allows operation of the same converter in the conventional buck regulator mode, so that the output can be regulated down to zero volts, allowing operation as a voltage regulating current limiting remote power controller (RPC). A major restriction on the use of the SCBBR technique is that it does not offer input / output isolation, but in the application discussed in this paper the TRF will provide the input / output isolation

The SCBBR concept was developed to provide high efficiency lightweight voltage regulation and provide a current limiting RPC function. The theory of operation, power stage circuit design, and performance of a proof of concept model are described in this paper.

\section{CIRCUIT OPERATION}

The operation of the circuit will be described by discussing the individual modes of operation, then describing the circuitry for each mode, and then showing how the circuits are combined. The converter operation is similar to an autotransformer in an AC system. Several taps or a variable transformer are used to increase or decrease the output voltage, and the kVA rating of the transformer is much smaller than the total power throughput.

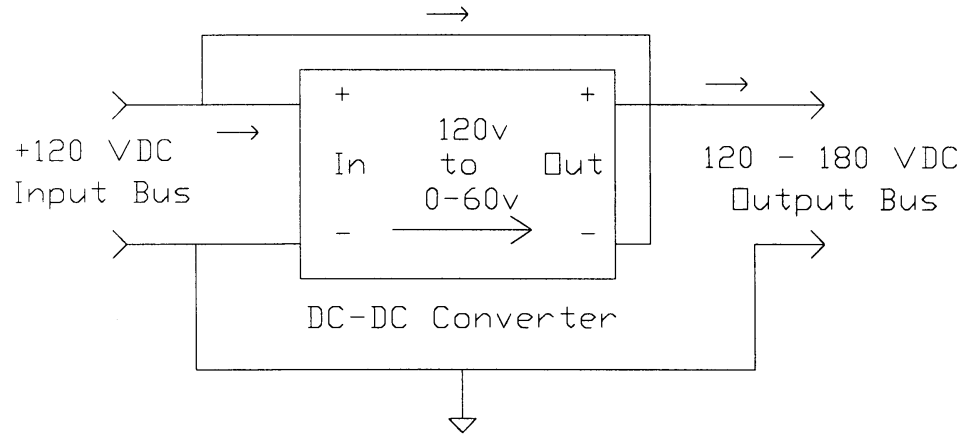

FIGURE 1. Boost Mode Power Flow Diagram.

The simplest mode is the boost mode, which will be described first. A diagram of the power flow is shown in Fig. 1. In the boost mode, the output of a low voltage converter is added in series with the input voltage to provide an output voltage equal to or greater than the input. In the boost mode, power from the converter is added to the output to increase the voltage. Most of the input power flows in the series path that goes through the output of the converter to the load connected to the output bus. Part of the input power goes to the DC-DC converter, where it is converted to a low voltage high current output that is added to the series path to boost the voltage. Only a portion of the power is processed by the DC-DC converter, so the converter size is small compared to the total output. Also, the overall efficiency is very high because there are no losses associated with the portion of the power that just passes thru the series path.

The buck mode of the SCBBR operates in a very different mode, using essentially the reverse of the boost mode operation. Because the buck mode operation is not as easy to understand as the boost mode, a battery analogy (Fig. 2) is presented to help explain the concept.

The boost mode battery analogy is shown on the left side of Fig. 2. Adding a battery in series with the input bus is similar to adding the output of the DC-DC converter in series with the input. The battery boosts the output voltage, but would become discharged during operation; a DC-DC converter allows continuous operation. 

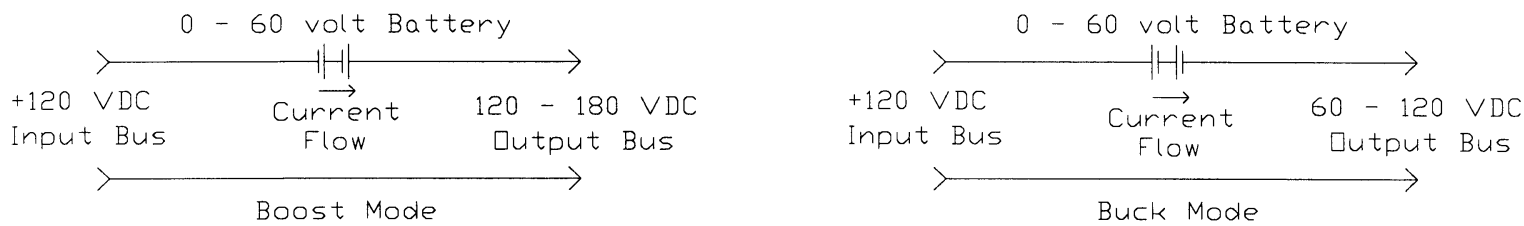

FIGURE 2. Series Battery Analogy.

The buck mode is shown on the right side of Fig. 2. The output voltage is reduced by the opposing voltage of the battery. Simply connecting a typical DC-DC converter (such as could be used for the boost mode) with the output reversed would not work though, because current cannot flow backwards thru the output of a typical DC-DC converter. Unlike in the boost mode case, the battery in the battery analogy of the buck mode is being charged. To use a DC-DC converter for continuous operation, he input power side of a DC-DC converter is connected in place of the battery, and the energy is returned to the input bus.

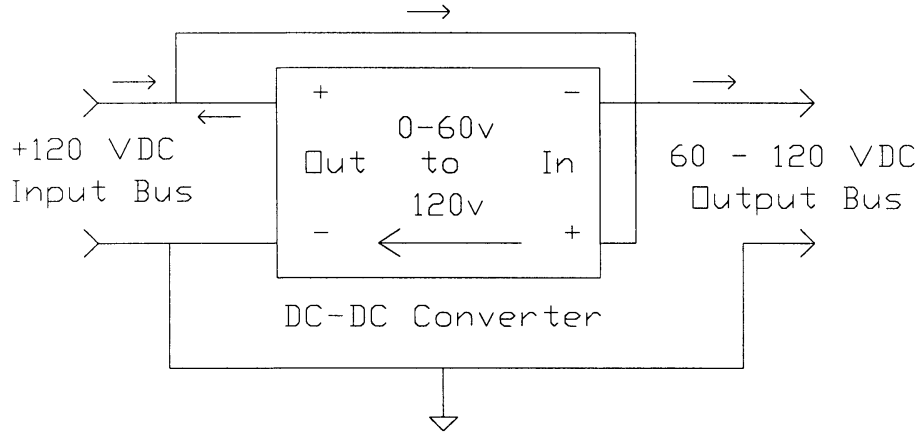

FIGURE 3. Buck Mode Power Flow Diagram.

The power flow diagram for the buck mode is shown in Fig. 3. The voltage drop across the input of the DC-DC converter reduces the SCBBR output voltage, and the power associated with this voltage drop is returned to the input bus by the DC-DC converter. The DC-DC converter used for the boost mode is conventional in that it operates with a moderately constant input voltage, and uses pulse width modulation (PWM) to vary the output voltage and control the boost. Conversely, the buck converter operates with a widely variable input voltage, including essentially zero, and outputs a relatively constant voltage. A large current, equal to the load current flows thru the power input side of the converter, independent of the amount of buck. Instead of a voltage fed converter configuration as used for the boost mode of operation, a current fed converter configuration is used for the buck mode.

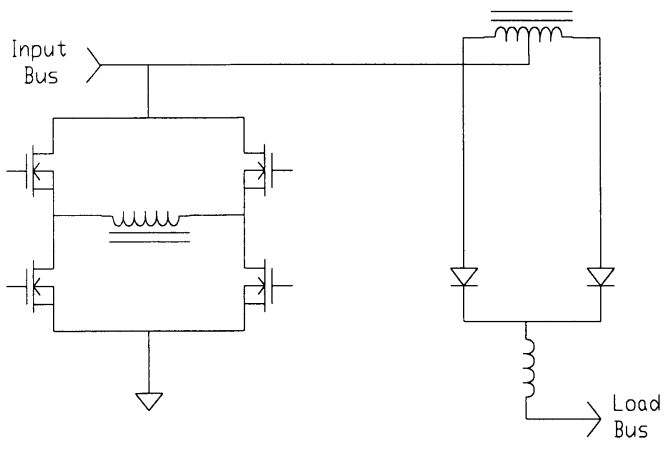

FIGURE 4. Boost Mode Schematic Diagram.
The SCBBR implementation of the boost mode uses a full bridge input stage and a center tapped transformer - rectifier output stage as shown in Fig. 4. When all the primary side switches are turned off there is no voltage across the transformer, and the input voltage is connected to the output thru the input filter, the secondary of the transformer, the rectifier diodes, and output filter. When the switches of the input bridge are conducting, a voltage is impressed on the secondary of the transformer. The instantaneous output at the rectifiers is the input voltage plus the transformer secondary voltage, which is equal to the input voltage divided by the transformer turns ratio. By varying the PWM duty cycle from 0 to $100 \%$ the average output can be controlled between 
a minimum of the input voltage, and a maximum of the input voltage plus the boost provided by the transformer secondary's output. The output filter smoothes the output and the average output voltage, ignoring conduction losses in the filters and rectifiers, will be:

$$
\mathrm{V}_{\text {out }}=\mathrm{V}_{\text {in }}+\mathrm{V}_{\text {in }} * \text { PWM Duty Cycle / Turns Ratio }
$$

The turns ratio for the prototype design is $2: 1$, allowing the output to be boosted up to $150 \%$ of the input.

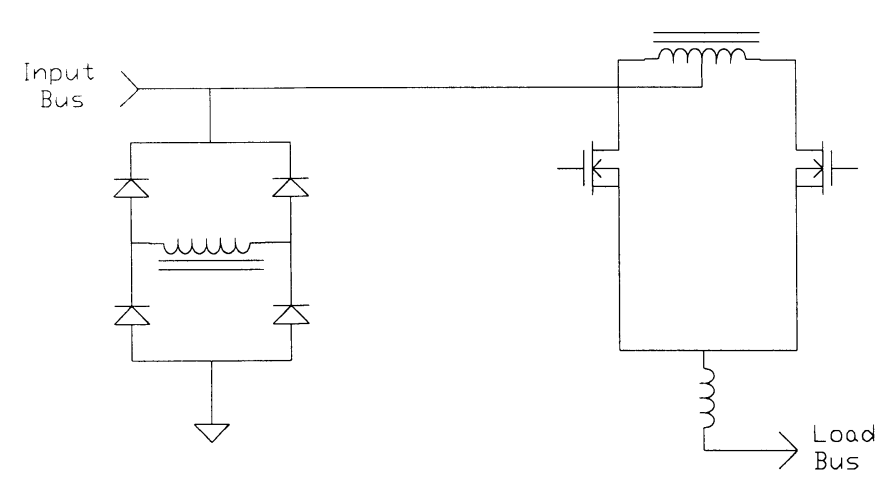

FIGURE 5. Buck Mode Schematic Diagram.

The schematic for buck mode operation is shown in Fig. 5. Many of the same components used for the boost converter are used for the buck converter, although in different roles. The center-tapped side of the transformer, which was the secondary in the boost mode, becomes the primary, and FET switches replace the rectifier diodes of the boost converter. (In the actual application the buck mode FETs are placed in series with the boost mode rectifiers.) The FETs that switched the primary of the transformer in the boost mode now function as a full wave bridge rectifier using the body diodes (or synchronous rectification with the FETs).

The output voltage of the DC-DC converter is recirculated back to the input bus of the SCBBR. Varying the duty cycle of the switches controls the ratio between the input and output voltage of the converter. Since the input bus voltage fixes the output voltage of the DC-DC converter, the effect of varying the duty cycle is to vary the voltage drop between the input bus and the SCBBR output voltage.

The switching action of the two switches in the primary of the buck converter might also be considered unusual in that either one or both switches are always turned on; they are never off simultaneously, even during the switching cycle. It is a current fed mode of operation. When both switches are conducting there is no voltage drop (except the small conduction losses) across the input of the DC-DC converter, so the output voltage of the SCBBR is equal to its input voltage. When one switch is open, the voltage across the transformer output will be equal to the SCBBR input bus voltage since the bridge rectifiers clamp it to that value. Therefore the input voltage of the converter will be equal to the input bus voltage divided by the transformer turns ratio. Varying the duty cycle controls the average voltage dropped across the DC-DC converter, and therefore the SCBBR output voltage.

The SCBBR output voltage in the buck mode can be computed as:

$$
\mathrm{V}_{\text {out }}=\mathrm{V}_{\text {in }}-\mathrm{V}_{\text {in }} * \text { PWM Duty Cycle / Turn Ratio, }
$$

where a PWM duty cycle of 0 corresponds to the switches being closed all the time and an duty cycle of $100 \%$ corresponds to each switch being closed 50\% of the time. Except for the sign in the equation, this equation is identical to the one for the boost mode. If the duty cycle is redefined such that the duty cycle for maximum buck is $-100 \%$, the equations become identical. As the PWM angle is varied from $-100 \%$ through 0 to $+100 \%$ the output of the SCBBR goes from full buck through no buck or boost up to full boost. For a 2:1 turns ratio transformer, the output can be varied from $50 \%$ to $150 \%$ of the input voltage. 


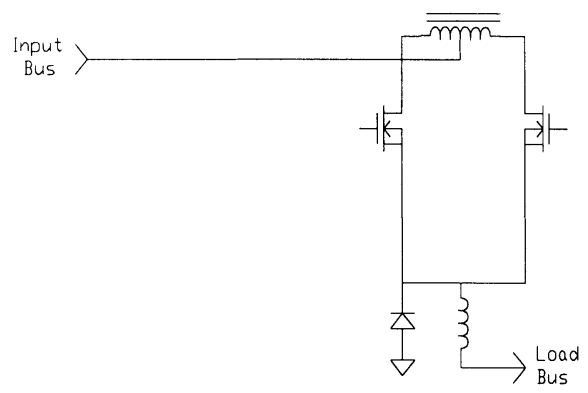

FIGURE 6. Current Limiting Mode Schematic Diagram.
The third mode of operation, the Current Limiting (CL) mode, is shown in Fig. 6. It uses many of the components used in the boost and buck modes, as well as some additional components. It is again a completely different mode of switching, and allows operation down to zero output voltage to allow an increased operating range, and particularly an OFF mode and turn-on / overload limiting function. In the CL mode the primary or full wave bridge side of the converter switches serve no purpose, and they are all tuned off. The FET switches used for the buck mode on the output bus side of the converter are switched simultaneously, effectively as one switch, and the transformer has no voltage across it. As shown in Fig. 6 a diode is added between the FET switches and common, and the FET switches, the diode, and the output inductor function as a conventional buck converter. The transformer has no function in this mode, and is in fact

shorted by an auxiliary FET (not shown) to reduce voltage transients.

In this paper this mode will be referred to as the CL mode to avoid confusion with the SCBBR buck mode previously described. In this mode the output voltage can be computed as:

$$
\mathrm{V}_{\text {out }}=\mathrm{V}_{\text {in }} * \text { PWM Angle }
$$

Although the form of this equation is quite different from the boost and buck mode equations given previously, the output voltage is still defined by the input voltage and PWM angle, and the output can be controlled between 0 volts and the input voltage. As the transformer is not used in this mode its turns ratio does not appear in the equation. The output voltage range in this mode overlaps completely the range in the SCBBR buck mode, but with lower efficiency, because it is switching all the power, and higher ripple currents in the filters. In the prototype the switching frequency is increased for operation in the CL mode to reduce the current ripple. The current limit mode is used only during turn on and overload conditions, or if the output voltage must be lower than that that can be obtained with the SCBBR buck mode. The CL mode with the PWM duty cycle $=0$ turns off the output, effectively a bus switch. Also, the equation is only valid for continuous conduction where the inductor current never goes to 0 .

It is important to note that the SCBBR input and output voltages are always positive and that the SCBBR input and output currents are always positive, except as noted below. However, the DC-DC converter within the SCBBR is bidirectional, and while the voltage on the input bus side is always positive and the current in the output bus side is always positive, but the current into the input bus side of the DC-DC converter itself is positive in the boost mode and negative in the buck mode, and the voltage at the output bus side of the dc-dc converter itself will be positive for the boost mode and negative for the buck mode.

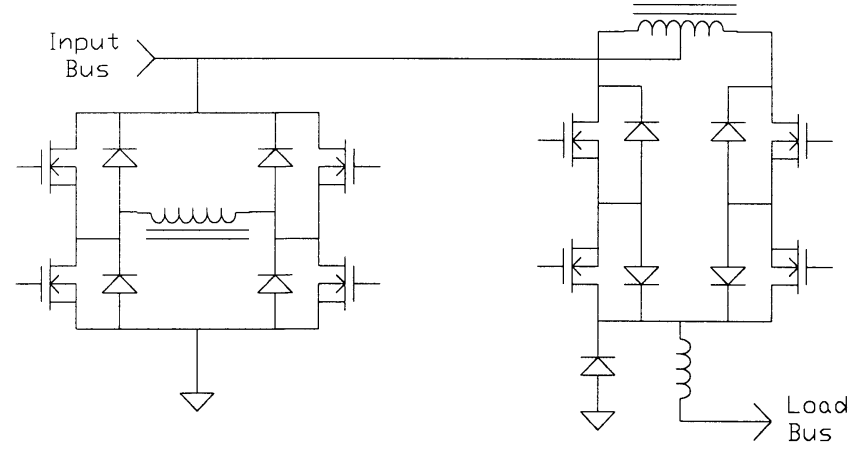

FIGURE 7. Complete SCBBR Schematic.
Essentially overlaying the boost, buck, and current limit mode schematics reveals the complete SCBBR / RPC schematic, as shown in Fig. 7.

This discussion has assumed that the input and output currents of the SCBBR are always positive. But if synchronous rectification is used on both sides of the DC-DC converter then current flow can be either direction. This is very useful because the magnetizing current of the transformer can be supplied from the input source, otherwise there would be a minimum output current, the current 
required to magnetize the transformer, below which the SCBBR buck mode would not operate. Switches that can conduct in both directions are already required on two of the switches on the SCBBR output. The logic to control the primary switches is straightforward, and provides the magnetizing current discussed above. By also using active switches in the other two positions of the output the efficiency will increase (synchronous rectification), and the SCBBR will operate in a regenerative mode wherein power is actually returned to the source from the load. The regenerative operation in the CL mode is possible if the free wheeling diode was replaced with a switch, but this has not been done in the prototype. This bilateral or regenerative operating mode can be useful for functions such as a battery charge discharge controller.

\section{ELECTRICAL EFFICIENCY}

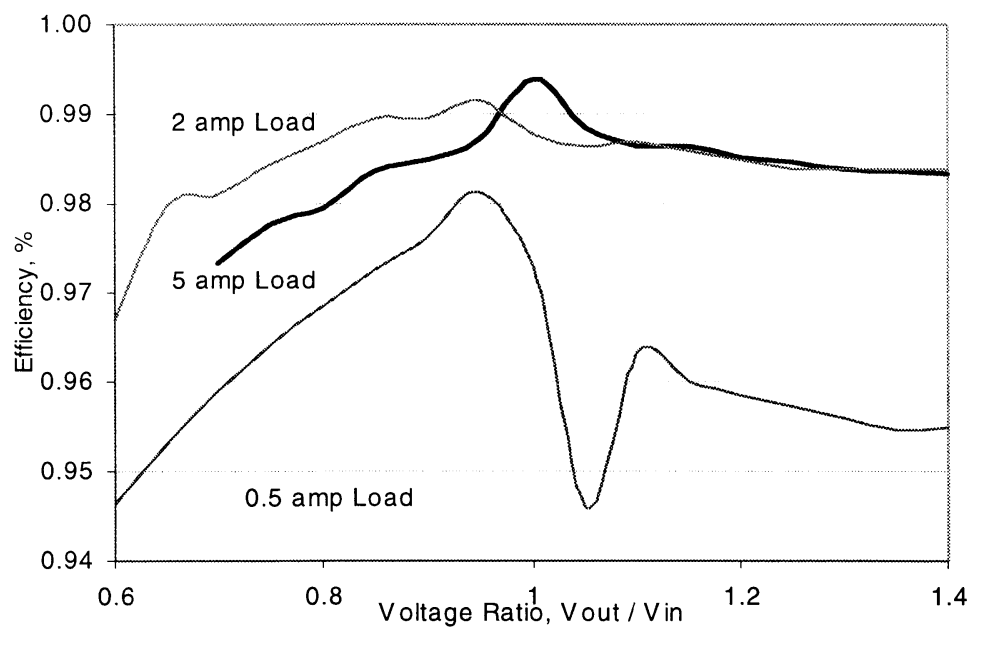

FIGURE 8. SCBBR Efficiency.
High electrical efficiency was the primary driver for developing the SCBBR technique; therefore the breadboard circuit was optimized for low losses. As such the design uses rather large semiconductors and magnetic components, and a relatively low $(25 \mathrm{kHz})$ switching frequency, but it clearly indicates the potential. Fig. 8 shows the efficiency as measured for a constant input voltage and constant load current as the buck or boost ratio is varied. Plots are shown for a 5-amp, a 2-amp, and a 0.5-amp load. The efficiency variations around a boost ratio of 1 is due to the overlap and the transition between the buck and boost modes. These plots show the high efficiency over a wide range of buck / boost ratios and load currents. The DC resistance of the series connected components (the input filter, the transformer secondary, the secondary switches, and the

output filter) is $.15 \mathrm{ohms}$, accounting for $.25 \%$ loss at the $2 \mathrm{amp}$ load, and $0.6 \%$ loss at 5 amp load. $2 / 3 \mathrm{rds}$ of the loss at full load is due to switching losses, magnetic components core losses, or resistive losses in the primary. Switching losses dominate at the 0.5 amp load but the efficiency is still above $95 \%$.

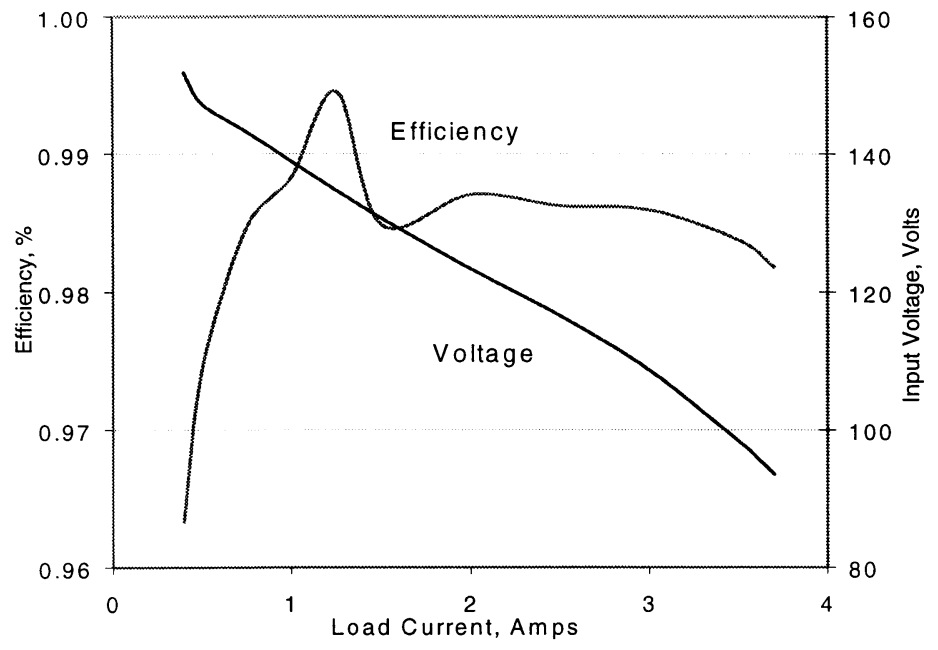

FIGURE 9. Efficiency as a Fuel Cell Regulator.
The current development of the SCBBR is for an application as a fuel cell regulator. The fuel cell application subjects the SCBBR to large input voltage variations. The target fuel cell is rated at 340 volts no load, and 200 volts at the full load current of $50 \mathrm{amps},(10 \mathrm{~kW})$. The intended load is a 270 -volt bus. The prototype SCBBR is being tested with a simulated fuel cell scaled at $50 \%$ of the full scale system voltage, 170 volts no load and 100 volts full load, and $10 \%$ of the full scale system current rating, 5 amps full load, working into a 135 volt bus. The input voltage variation and SCBBR efficiency is shown in Fig. 9. The efficiency doesn't fall badly until about $10 \%$ load. The power loss is a relatively constant 2 to 3 watts below $50 \%$ 
power due to switching losses. These efficiencies are for the power stage only; the control power requirement of the prototype, including gate drive, is 3 to 4 watts.

\section{VOLTAGE REGULATION AND STABILITY}

Steady state voltage regulation in a converter is primarily a measure of the performance of the voltage regulation feedback loop. The regulation can be as good as the regulator, independent of the transfer characteristics of the converter. However, the preceding discussions discussed the transfer functions of this converter and the similarity of them throughout the three different modes, and implied that the output voltage could be determined based on the

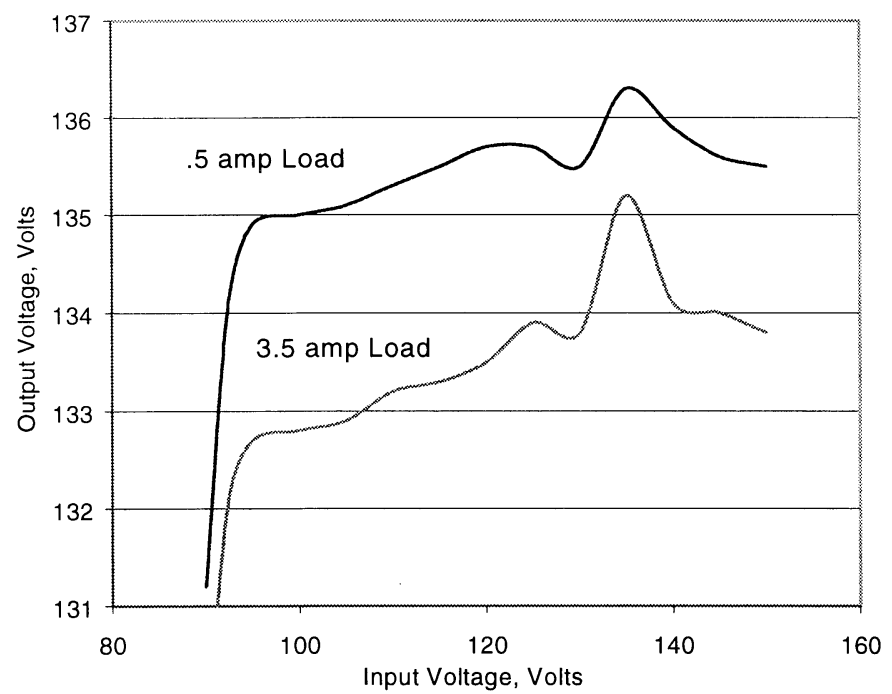

Figure 10. Open loop Voltage Regulation input voltage, the transformer ratio, and the PWM angle. Fig. 10 shows the open loop regulation of the SCBBR as a function of input voltage and output current. The open loop regulation generally continues into the current limit mode also, but not as accurately, and only the start of this region is shown in the figure. A closed loop regulator is also included on the prototype SCBBR, but its requirements are minimal since it only function is to trim out the remaining error shown in the figure. Its control range is limited to altering the output voltage a few percent, and it uses only the integral of the voltage error. Transient response, and damping, is provided by the open loop path, which simplifies the design of the controller path. In fact only an integral control loop was used in the prototype with no stability problems.

\section{Transient Overload Response}

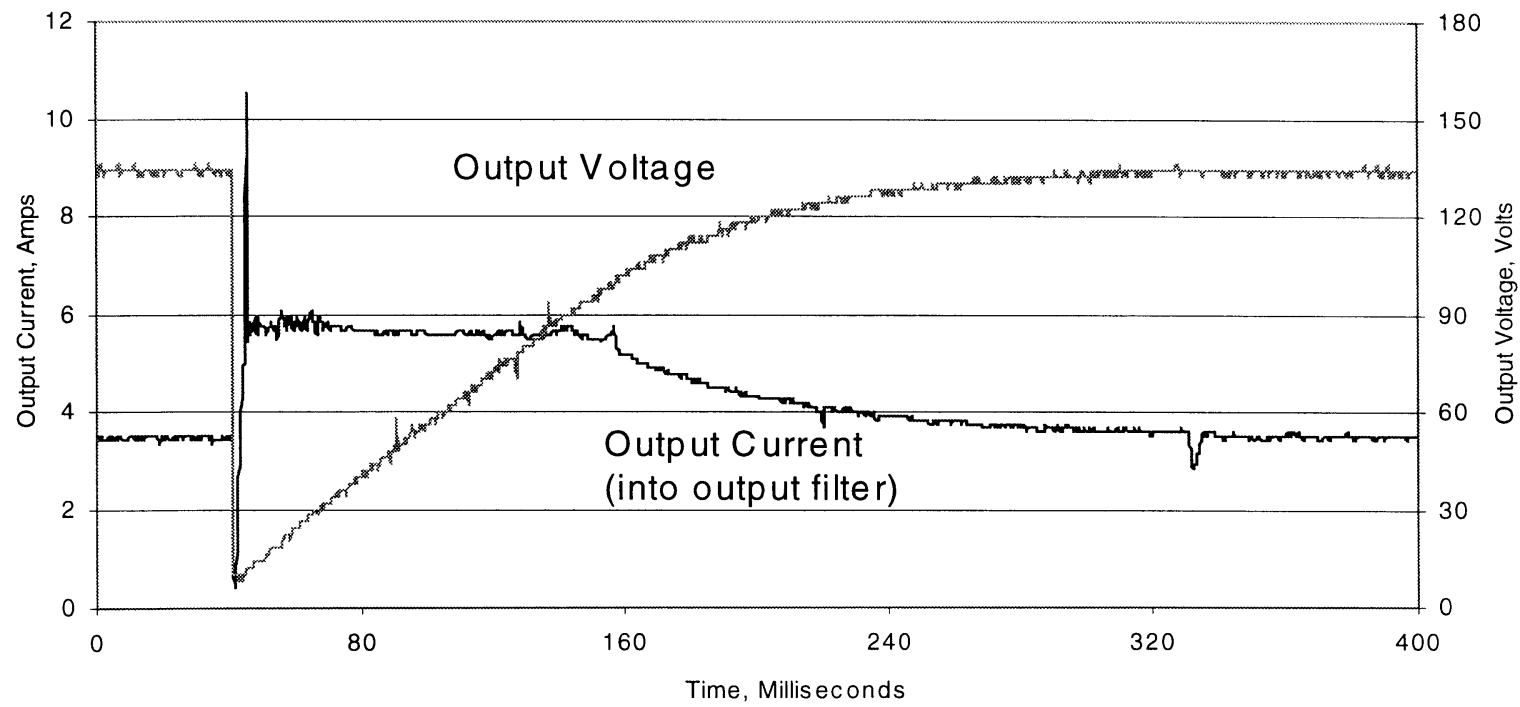

FIGURE 11. Transient Overload Response. 
As discussed so far, the SCBBR is a stiff voltage source. To be useful in a real system the ability to limit currents during startup and into an overload is required. A stiff voltage source characteristic is desirable for regulating voltage, but the low impedance would result in large currents in the event of an overload. Two control circuit functions were added to control the overload currents, and startup. The first is an instantaneous overcurrent sense at twice rated current to shut off all switches and limit the peak current into the output filter during startup or the initial application of a severe overload. The second is a linear proportional plus integral current regulation loop at 1.5 times rated current to control the PWM angle until the output voltage returns to the operating range and the voltage regulator takes over. Implementation of these loops was successful as illustrated in Fig. 11, which shows the recovery from applying a large capacitor as a transient load while running at rated current. The capacitor is 100 times the output filter capacitance so the output voltage collapses almost completely. The instantaneous overcurrent sensing limits the initial spike of current (into the filter), and then the SCBBR runs under current limit until the capacitor is charged. The current sensing and the data plotted are the current into the output filter. This is the same as the currents in the output switches, and it is the critical current to be controlled for protection of the SCBBR.

\section{SUMMARY}

The boost implementation of partial power processing DC-DC converters has been shown to be an effective method to provide efficient light-weight voltage regulation. The SCBBR concept has been developed to implement both the boost and a buck mode of regulation to enhance the regulation range over the boost only implementation. Current limiting Remote Power Controller (RPC) capabilities were also added, and a breadboard was developed to demonstrate the potential capabilities of the concept. The breadboard operates at over $98 \%$ power stage efficiency over a broad input - output voltage and load range, and provides fault protection, overcurrent limiting, and bus switching. A $10 \mathrm{~kW}$ version is under development to provide high voltage DC bus regulation and switching for high power aerospace applications.

\section{REFERENCES}

Mason, Lee. , "A Power Conversion Concept for the Jupiter Icy Moon Orbiter,” First AIAA/ASME/IEEE International Energy Engineering Conference, AIAA-2003-6007, 2003.

Button, Robert M., "A Modular PMAD System for Small Spacecraft," NASA Technical Memorandum 1998-206628, NASA Lewis Research Center, Cleveland, OH., 1998.

Button, Robert M., "An Advanced Photovoltaic Array Regulator Module," NASA Technical Memorandum 107304, NASA Lewis Research Center, Cleveland, OH., 1996. 